Document downloaded from:

http://hdl.handle.net/10251/64564

This paper must be cited as:

Liu, X.; Qin, X.; Benítez López, J. (2015). Some additive results on Drazin inverse. Applied Mathematics - A Journal of Chinese Universities. 30(4):479-490. doi:10.1007/s11766-0153333-4.

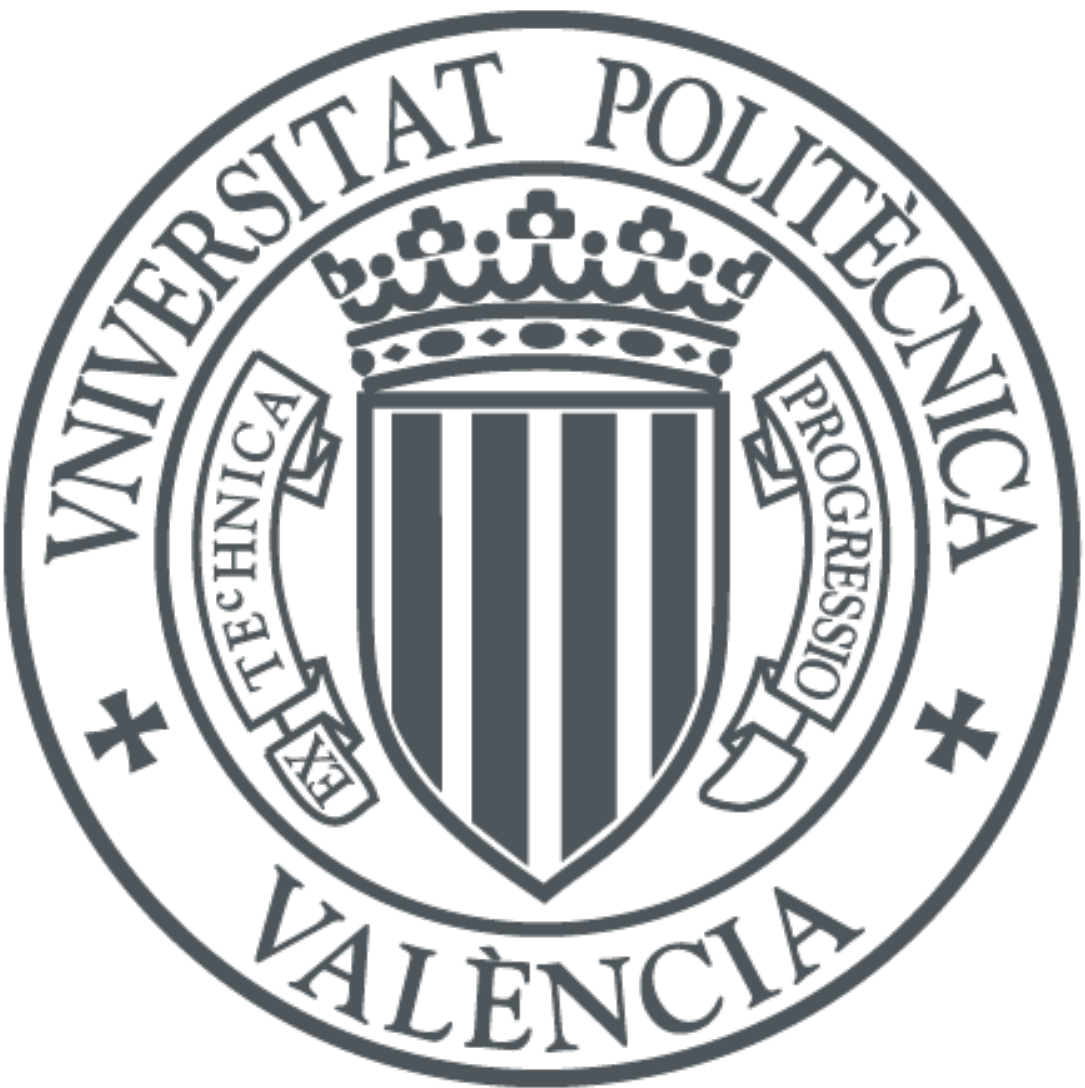

The final publication is available at

http://dx.doi.org/10.1007/s11766-015-3333-4

Copyright Springer Verlag (Germany)

Additional Information 


\title{
Some additive results on Drazin inverse
}

\author{
Xiaoji Liu, Xiaolan Qin, Julio Benítez
}

\begin{abstract}
In this paper, we investigate additive results of the Drazin inverse of elements in a ring $\mathcal{R}$. Under the condition $a b=b a$, we show that $a+b$ is Drazin invertible if and only if $a a^{D}(a+b)$ is Drazin invertible, where the superscript $D$ means the Drazin inverse. Furthermore we find an expression of $(a+b)^{D}$. As an application we give some new representations for the Drazin inverse of a $2 \times 2$ block matrix.
\end{abstract}

\section{$\S 1 \quad$ Introduction and previous results}

In this paper, $\mathcal{R}$ will denote a unital ring whose unity is $\mathbb{1}$. Let us recall that an element $a \in \mathcal{R}$ has a Drazin inverse [18] if there exists $b \in \mathcal{R}$ such that

$$
b a b=b, \quad a b=b a, \quad a-a^{2} b \text { is nilpotent. }
$$

The element $b$ above is unique if it exists and is denoted by $a^{D}$. The nilpotency index of $a-a^{2} a^{D}$ is called the Drazin index of $a$, denoted by ind $(a)$. The notation $a^{\pi}$ means $\mathbb{1}-a a^{D}$ for any Drazin invertible element $a \in \mathcal{R}$. Observe that by the definition of the Drazin inverse, $a a^{\pi}$ is nilpotent. The subset of $\mathcal{R}$ composed of Drazin invertible elements will be denote by $\mathcal{R}^{D}$.

Drazin proved, [18], that if $a, b \in \mathcal{R}^{D}$ and $a b=b a=0$, then $a+b \in \mathcal{R}^{D}$ and $(a+b)^{D}=$ $a^{D}+b^{D}$. In recent years, many papers focused on the problem under some weaker conditions. Hartwig et al., [19], expressed $(A+B)^{D}$ under the one-side condition $A B=0$, where $A$ and $B$ are complex square matrices. This result was extended to bounded linear operators on an arbitrary complex Banach space by Djordjević and Wei in [15]. Again, it was extended for morphisms on arbitrary additive categories by Chen et al. in [8]. More results on the Drazin inverse or the generalized Drazin inverse can also be found in $[3,5,6,8,9,11,12,15]$. In particular we must cite [13]: in this paper, the authors, under the commutative condition of $A B=B A$ (when $A$ and $B$ are Drazin invertible linear operators in Banach spaces), gave explicit representations of $(A+B)^{D}$ in term of $A, A^{D}, B$, and $B^{D}$. 
In this paper, we assume that $a$ and $b$ are Drazin invertible elements which satisfy $a b=b a$ or $a^{\pi} b=0$ and $a^{n} b=b a^{n}$ for some $n \in \mathbb{N}$, and we conclude that $a+b$ is Drazin invertible if and only if $a a^{D}(a+b)$ is Drazin invertible. Also we obtain an explicit expression for $(a+b)^{D}$. As an application, we give additive results of block matrices under some conditions.

We give now some previous results which will be useful in proving our results.

Lemma 1.1. Let $a, x \in \mathcal{R}$. If $a x=x a$ and there exists $n \in \mathbb{N}$ such that $a^{n}=0$, then $\mathbb{1}-x a$ is invertible and $(\mathbb{1}-x a)^{-1}=\sum_{i=0}^{n-1} x^{i} a^{i}$.

Proof. Let $y=\sum_{i=0}^{n-1} x^{i} a^{i}$. It is enough to verify $(\mathbb{1}-x a) y=y(\mathbb{1}-x a)=\mathbb{1}$.

Lemma 1.2. Let $x, y$ be two commuting nilpotent elements of $\mathcal{R}$. Then $x+y$ is nilpotent.

Proof. It is enough to recall $(x+y)^{n}=\sum_{k=0}^{n}\left(\begin{array}{l}n \\ k\end{array}\right) x^{k} y^{n-k}$ for any $n \in \mathbb{N}$ since $x y=y x$.

Next theorem was proved by Drazin [18, Th. 1].

Theorem 1.1. Let $a \in \mathcal{R}^{D}$ and $b \in \mathcal{R}$. If $a b=b a$, then $a^{D} b=b a^{D}$.

\section{$\S 2$ Main results}

Let us observe the expression for $(a-b)^{D}$ in [24, Th. 2.3]. If we assume that $w=a a^{D}(a+b)$ instead of $w=a a^{D}(a-b) b b^{D}$, we will get a much simpler expression for $(a+b)^{D}$.

Theorem 2.1. Let $a, b \in \mathcal{R}$ be Drazin invertible. If $a b=b a$, then $w=a a^{D}(a+b)$ is Drazin invertible if and only if $a+b$ is Drazin invertible. In this case, we have

$$
(a+b)^{D}=w^{D}+a^{\pi}\left(\mathbb{1}+b^{D} a a^{\pi}\right)^{-1} b^{D}=w^{D}+a^{\pi}\left(\sum_{i=0}^{\operatorname{ind}(a)-1}\left(-b^{D} a\right)^{i}\right) b^{D} .
$$

Proof. Recall that $a a^{\pi}$ is nilpotent and its index of nilpotency is the Drazin index of $a$. Let $r=\operatorname{ind}(a)$. Since $a b=b a$, by Theorem 1.1, $a^{D} b=b a^{D}$ and $a b^{D}=b^{D} a$. From $a^{D} b=b a^{D}$ we obtain $a^{\pi} b=b a^{\pi}$. Again by Theorem 1.1, $a^{\pi}$ commutes with $b^{D}$. Therefore, $b^{D} a^{\pi} a=a^{\pi} a b^{D}$. By Lemma 1.1 we get that $\mathbb{1}+b^{D} a a^{\pi}$ is invertible and

$$
\left(\mathbb{1}+b^{D} a a^{\pi}\right)^{-1}=\sum_{i=0}^{r-1}\left(-b^{D} a a^{\pi}\right)^{i}=\mathbb{1}+a^{\pi} \sum_{i=1}^{r-1}\left(-b^{D} a\right)^{i} .
$$

In the rest of the proof, we will use frequently that $\left\{\mathbb{1}, a, b, a^{D}, b^{D}\right\}$ is a commutative family.

Assume that $w$ is Drazin invertible and let us define

$$
x=w^{D}+a^{\pi}\left(\mathbb{1}+b^{D} a a^{\pi}\right)^{-1} b^{D} .
$$

From $a b=b a$ and $a^{D} b=b a^{D}$, we have $w(a+b)=a a^{D}(a+b)(a+b)=(a+b) w$. By Theorem 1.1, we obtain $w^{D}(a+b)=(a+b) w^{D}$. Since $r=\operatorname{ind}(a)$, then $\left(a a^{\pi}\right)^{r}=0$, or equivalently, 
$a^{r} a^{\pi}=0$. We get

$$
\begin{aligned}
& (a+b) a^{\pi}\left(\mathbb{1}+b^{D} a a^{\pi}\right)^{-1} b^{D} \\
& =(a+b)\left[\mathbb{1}+\left(-b^{D} a\right) a^{\pi}+\left(-b^{D} a\right)^{2} a^{\pi}+\cdots+\left(-b^{D} a\right)^{r-1} a^{\pi}\right] b^{D} a^{\pi} \\
& =(a+b)\left[\mathbb{1}+\left(-b^{D} a\right)+\left(-b^{D} a\right)^{2}+\cdots+\left(-b^{D} a\right)^{r-1}\right] b^{D} a^{\pi} \\
& \left.=\left[a b^{D}+a\left(-b^{D} a\right) b^{D}+a\left(-b^{D} a\right)^{2} b^{D}+\cdots+a\left(-b^{D} a\right)^{r-1} b^{D}\right)\right] a^{\pi} \\
& \quad+\left[b b^{D}+b\left(-b^{D} a\right) b^{D}+b\left(-b^{D} a\right)^{2} b^{D}+\cdots+b\left(-b^{D} a\right)^{r-1} b^{D}\right] a^{\pi} \\
& =\left[a b^{D}-\left(a b^{D}\right)^{2}+\left(a b^{D}\right)^{3}+\cdots+(-1)^{r-2}\left(a b^{D}\right)^{r-1}+(-1)^{r-1}\left(a b^{D}\right)^{r}\right] a^{\pi} \\
& \quad+\left[b b^{D}-a b^{D}+\left(a b^{D}\right)^{2}+\cdots+(-1)^{r-1}\left(a b^{D}\right)^{r-1}\right] a^{\pi} \\
& =b b^{D} a^{\pi} .
\end{aligned}
$$

So, we get

$$
(a+b) x=(a+b)\left(w^{D}+a^{\pi}\left(\mathbb{1}+b^{D} a a^{\pi}\right)^{-1} b^{D}\right)=(a+b) w^{D}+b b^{D} a^{\pi} .
$$

Since $\left\{\mathbb{1}, a, b, a^{D}, b^{D}, w, w^{D}\right\}$ is a commutative family, we get $x(a+b)=(a+b) x$.

Next, we give the proof of $x(a+b) x=x$. From (2) we can write $(a+b) x=x^{\prime}+x^{\prime \prime}$, where $x^{\prime}=w^{D}(a+b)$ and $x^{\prime \prime}=b^{D} b a^{\pi}$. Observe that

$$
w+a^{\pi}(a+b)=a a^{D}(a+b)+\left(\mathbb{1}-a a^{D}\right)(a+b)=a+b .
$$

From $w a^{\pi}=(a+b) a a^{D} a^{\pi}=0$ we get $w^{D} a^{\pi}=\left(w^{D}\right)^{2} w a^{\pi}=0$, hence

$$
\begin{aligned}
x x^{\prime} & =\left(w^{D}+a^{\pi}\left(\mathbb{1}+b^{D} a a^{\pi}\right)^{-1} b^{D}\right) w^{D}(a+b) \\
& =\left(w^{D}\right)^{2}(a+b)=w^{D}(a+b) w^{D}=w^{D}\left(w+a^{\pi}(a+b)\right) w^{D}=w^{D}
\end{aligned}
$$

and

$$
\begin{aligned}
x x^{\prime \prime} & =\left(w^{D}+a^{\pi}\left(\mathbb{1}+b^{D} a a^{\pi}\right)^{-1} b^{D}\right) b^{D} b a^{\pi} \\
& =\left(a^{\pi}\left(\mathbb{1}+b^{D} a a^{\pi}\right)^{-1} b^{D}\right) b^{D} b a^{\pi} \\
& =\left(\mathbb{1}+b^{D} a a^{\pi}\right)^{-1} b^{D} a^{\pi} \\
& =x-w^{D} .
\end{aligned}
$$

So, we get $x(a+b) x=x\left(x^{\prime}+x^{\prime \prime}\right)=x$.

Now we will prove that $(a+b)-(a+b)^{2} x$ is nilpotent. Since $a+b=w+a^{\pi}(a+b), a^{\pi} w=0$, and $a^{\pi} w^{D}=0$, we have

$$
\begin{aligned}
(a+b)^{2} w^{D} & =\left(w+a^{\pi}(a+b)\right)^{2} w^{D} \\
& =\left(w^{2}+2 w a^{\pi}(a+b)+a^{\pi}(a+b)^{2}\right) w^{D}=w^{2} w^{D}=w-w w^{\pi} .
\end{aligned}
$$

Also we have

$$
(a+b) b^{D} b a^{\pi}=(a+b) a^{\pi}\left(\mathbb{1}-b^{\pi}\right)=a a^{\pi}+b a^{\pi}-a a^{\pi} b^{\pi}-a^{\pi} b b^{\pi} .
$$


From (2), (3), and (4) we get

$$
\begin{aligned}
& (a+b)-(a+b)^{2} x \\
& =(a+b)-(a+b)\left(w^{D}(a+b)+b b^{D} a^{\pi}\right) \\
& =(a+b)-\left(w-w w^{\pi}+a a^{\pi}+b a^{\pi}-a a^{\pi} b^{\pi}-a^{\pi} b b^{\pi}\right) \\
& =(a+b)-\left[(a+b) a a^{D}+(a+b) a^{\pi}-a a^{\pi} b^{\pi}-a^{\pi} b b^{\pi}-w w^{\pi}\right] \\
& =(a+b)-\left[(a+b)-a a^{\pi} b^{\pi}-a^{\pi} b b^{\pi}-w w^{\pi}\right] \\
& =a a^{\pi} b^{\pi}+a^{\pi} b b^{\pi}+w w^{\pi} .
\end{aligned}
$$

Since $a a^{\pi}, b b^{\pi}$, and $w w^{\pi}$ are nilpotent, and $\left\{a a^{\pi}, b b^{\pi}, w w^{\pi}\right\}$ is a commuting family, then by using Lemma 1.2 we get the nilpotency of $(a+b)-(a+b)^{2} x$. Therefore, we have proved $a+b \in \mathcal{R}^{D}$ and $(a+b)^{D}=x$, i.e., the expression (1).

Conversely, let us assume $a+b \in \mathcal{R}^{D}$. Let $y=a a^{D}(a+b)^{D}$. We will prove that $w=$ $a a^{D}(a+b) \in \mathcal{R}^{D}$ and $w^{D}=y$. Observe that Theorem 1.1 implies that $\left\{a, b, a^{D}, b^{D},(a+b)^{D}\right\}$ is a commuting family. Now, having in mind $\left(a a^{D}\right)^{2}=a a^{D}$, it is simple to prove $w y=y w=$ $a a^{D}(a+b)(a+b)^{D}, y^{2} w=y$, and $w^{2} y-w=a a^{D}\left[(a+b)^{2}(a+b)^{D}-(a+b)\right]$, which leads to the nilpotency of $w^{2} y-w$. The proof is finished.

Corollary 2.1. Let $a, b \in \mathcal{R}$ be Drazin invertible. If $a b=b a$ and $b a a^{\pi}=0$, then $w=a a^{D}(a+b)$ is Drazin invertible if and only if $a+b$ is Drazin invertible. In this case, we have

$$
(a+b)^{D}=w^{D}+a^{\pi} b^{D} .
$$

Proof. From $b a a^{\pi}=0$, we have $b^{D} a a^{\pi}=\left(b^{D}\right)^{2} b a a^{\pi}=0$. It is enough apply Theorem 2.1 to prove this corollary.

Theorem 2.2. Let $a, b \in \mathcal{R}$ be Drazin invertible, $a^{\pi} b=0$ and $a^{n} b=b a^{n}$ for some $n \in \mathbb{N}$. Then $a+b$ is Drazin invertible if and only if $w=a a^{D}(a+b)$ is Drazin invertible. In this case, we have

$$
(a+b)^{D}=w^{D} .
$$

Proof. From $a \in \mathcal{R}^{D}$, it is simple to prove that $a^{n} \in \mathcal{R}^{D}$ and $\left(a^{n}\right)^{D}=\left(a^{D}\right)^{n}$. In addition, $\left(a^{n}\right)^{\pi}=\mathbb{1}-a^{n}\left(a^{n}\right)^{D}=\mathbb{1}-\left(a a^{D}\right)^{n}=\mathbb{1}-a a^{D}=a^{\pi}$. Since $a^{n} b=b a^{n}$, by Theorem 1.1 we get $\left(a^{n}\right)^{D} b=b\left(a^{n}\right)^{D}$, and therefore, $a^{\pi} b=b a^{\pi}$ and $a a^{D} b=b a a^{D}$. Also, the following equality will be useful:

$$
w+a^{\pi}(a+b)=a a^{D}(a+b)+\left(\mathbb{1}-a a^{D}\right)(a+b)=a+b .
$$

Since $a a^{D}$ commutes with $a$ and $b$, we get $w a^{\pi}=a^{\pi} w=0$.

Assume that $w$ is Drazin invertible. We will prove that $w^{D}$ is the Drazin inverse of $a+b$, i.e., we will prove $w^{D}(a+b)=(a+b) w^{D},\left(w^{D}\right)^{2}(a+b)=w^{D}$, and $(a+b)^{2}-w^{D}$ is nilpotent.

Since $a a^{D} b=b a a^{D}$, we get

$$
w(a+b)=a a^{D}(a+b)(a+b)=(a+b) a a^{D}(a+b)=(a+b) w .
$$

By Theorem 1.1 we obtain $w^{D}(a+b)=(a+b) w^{D}$.

From $w a^{\pi}=0$ we get $w^{D} a^{\pi}=\left(w^{D}\right)^{2} w a^{\pi}=0$. By using $w^{D} a^{\pi}=0$ and (5) we have

$$
\left(w^{D}\right)^{2}(a+b)=\left(w^{D}\right)^{2}\left(w+a^{\pi}(a+b)\right)=\left(w^{D}\right)^{2} w+\left(w^{D}\right)^{2} a^{\pi}(a+b)=w^{D} .
$$


Since $a+b=w+a^{\pi}(a+b)$ and $a^{\pi} w=w a^{\pi}=0$, we have

$$
(a+b)^{2}=\left(w+a^{\pi}(a+b)\right)^{2}=w^{2}+a^{\pi}(a+b)^{2} .
$$

Hence from $a^{\pi} w^{D}=a^{\pi} w\left(w^{D}\right)^{2}=0$ we obtain

$$
\begin{aligned}
(a+b)^{2} w^{D} & =\left(w^{2}+a^{\pi}(a+b)^{2}\right) w^{D}=w^{2} w^{D}=w-w w^{\pi} \\
& =a a^{D}(a+b)-w w^{\pi}=\left(\mathbb{1}-a^{\pi}\right)(a+b)-w w^{\pi} \\
& =a+b-a^{\pi} a-a^{\pi} b-w w^{\pi} .
\end{aligned}
$$

From $a^{\pi} b=0$, we have $a+b-(a+b)^{2} w^{D}=a^{\pi} a+w^{\pi} w$.

From $a^{\pi} w=w a^{\pi}$, we have $a^{\pi} w^{D}=w^{D} a^{\pi}$, so, we get

$$
a^{\pi} w^{\pi}=a^{\pi}\left(\mathbb{1}-w w^{D}\right)=\left(\mathbb{1}-w w^{D}\right) a^{\pi}=w^{\pi} a^{\pi} .
$$

From $w a^{\pi}=a^{\pi} w=0$ we obtain $\left(a a^{\pi}\right)\left(w w^{\pi}\right)=0$ and $\left(w w^{\pi}\right)\left(a a^{\pi}\right)=0$. Hence for any $k \in \mathbb{N}$ we have

$$
\left(a+b-(a+b)^{2} w^{D}\right)^{k}=\left(a^{\pi} a+w^{\pi} w\right)^{k}=\left(a^{\pi} a\right)^{k}+\left(w^{\pi} w\right)^{k} .
$$

Since $a a^{\pi}$ and $w w^{\pi}$ are nilpotent, it follows that $(a+b)-(a+b)^{2} w^{D}$ is nilpotent. We have just proved that $a+b \in \mathcal{R}^{D}$ and $(a+b)^{D}=w^{D}$.

Assume that $a+b \in \mathcal{R}^{D}$. We will prove that $w=a a^{D}(a+b) \in \mathcal{R}^{D}$ and the Drazin inverse of $a+b$ is $w^{D}$, i.e., $(a+b)^{D} w=w(a+b)^{D},\left((a+b)^{D}\right)^{2} w=(a+b)^{D}$, and $w^{2}(a+b)^{D}-w$ is nilpotent.

Since $a a^{D}$ commutes with $a$ and $b$ we have $(a+b) w=w(a+b)$. By Theorem 1.1, one gets $(a+b) w^{D}=w^{D}(a+b)$.

Since $a$ is Drazin invertible, we can write $a=a_{1}+a_{2}$ (this is the core-nilpotent decomposition of $a$, see e.g [16, Ch. 2]), where $a_{1} \in a a^{D} \mathcal{R} a a^{D}$ and $a_{2} \in a^{\pi} \mathcal{R} a^{\pi}$ is nilpotent. From $a^{\pi} b=$ $b a^{\pi}=0$ we obtain $b \in a a^{D} \mathcal{R} a a^{D}$. Hence $a+b$ can be decomposed as

$$
a+b=\left(a_{1}+b\right)+a_{2}, \quad a_{1}+b \in a a^{D} \mathcal{R} a a^{D}, a_{2} \in a^{\pi} \mathcal{R} a^{\pi} .
$$

From $(a+b) a a^{D}=a a^{D}(a+b)$ and Theorem 1.1 we get $(a+b)^{D} a a^{D}=a a^{D}(a+b)^{D}$, and therefore,

$$
(a+b)^{D}=a a^{D}(a+b)^{D} a a^{D}+a a^{D}(a+b)^{D} a^{\pi}+a^{\pi}(a+b)^{D} a a^{D}+a^{\pi}(a+b)^{D} a^{\pi}
$$

can be also decomposed as

$$
(a+b)^{D}=u+v, \quad u \in a a^{D} \mathcal{R} a a^{D}, v \in a^{\pi} \mathcal{R} a^{\pi} .
$$

From the definition of the Drazin inverse and (6), (7) we have that $a_{1}+b, a_{2} \in \mathcal{R}^{D}$ and $\left(a_{1}+b\right)^{D}=u, a_{2}^{D}=v$. But, $a_{2}^{D}=0$ because $a_{2}$ is nilpotent. Therefore, $(a+b)^{D}=\left(a_{1}+b\right)^{D} \in$ $a a^{D} \mathcal{R} a a^{D}$. Now

$$
\begin{aligned}
\left((a+b)^{D}\right)^{2} w & =\left(\left(a_{1}+b\right)^{D}\right)^{2} a a^{D}(a+b) \\
& =\left(\left(a_{1}+b\right)^{D}\right)^{2}(a+b)=\left((a+b)^{D}\right)^{2}(a+b)=(a+b)^{D} .
\end{aligned}
$$

Now, let us prove that $w^{2}(a+b)^{D}-w$ is nilpotent. We have proved that $a a^{D}$ commutes 
with $a+b$. Since $a a^{D}$ is an idempotent,

$$
\begin{aligned}
w^{2}(a+b)^{D}-w & =\left[a a^{D}(a+b)\right]^{2}(a+b)^{D}-a a^{D}(a+b) \\
& =a a^{D}(a+b)^{2}(a+b)^{D}-a a^{D}(a+b) \\
& =a a^{D}\left[(a+b)^{2}(a+b)^{D}-(a+b)\right] .
\end{aligned}
$$

Since $a a^{D}$ commutes with $a+b$ and $(a+b)^{D}$, and $(a+b)^{2}(a+b)^{D}-(a+b)$ is nilpotent, then $w^{2}(a+b)^{D}-w$ is nilpotent. Therefore, $w \in \mathcal{R}^{D}$ and $w^{D}=(a+b)^{D}$. The proof is finished.

If $(\mathcal{R}, \cdot)$ is a ring with a unity $\mathbb{1}$, then we can define a new multiplication in $\mathcal{R}$ by $a \odot b=$ $b a$. With this multiplication, $(\mathcal{R}, \odot)$ becomes a ring with the same unity $\mathbb{1}$. We can apply Theorem 2.2 to $(\mathcal{R}, \odot)$ obtaining a dual result.

\section{$\S 3$ Applications}

In this section, we give some formulas for the Drazin inverse of a $2 \times 2$ block matrix under some conditions. Let $\mathbb{C}^{m \times n}$ be the set of all the $m \times n$ matrices over the complex field.

Let $M$ be a matrix of the form

$$
M=\left[\begin{array}{cc}
A & B \\
C & D
\end{array}\right], \quad A \in \mathbb{C}^{m \times m}, D \in \mathbb{C}^{n \times n} .
$$

Campbell and Meyer, [2, Ch. 7] proposed the (until now open) problem to find an explicit formula of the Drazin inverse of $M$ in terms of the blocks of $M$. Several authors have investigated this problem and they were able to find some partial answers (imposing some conditions on the blocks of $M)$. Here we write an exemplary list.

- $B=0$ (or $C=0)$. See $[2$, Ch. 7] or $[23]$.

- $B C=0, D C=0$ (or $B D=0)$, and $D$ is nilpotent. See [20].

- $B C A=0, B D=0$, and $D C=0$ (or $B C$ is nilpotent). See [4].

- $B C A=0, B C B=0, D C A=0$, and $D C B=0$. See [25].

- $B C=0, B D=0$ and $D C=0$. See [14].

- $B C=0$ and $D C=0$. See $[10]$.

- $B C A=0, B C B=0, A B D=0$, and $C B D=0$. See [22];

- $B C=0$ and $B D=0$. See [17].

We will find several expressions for $M^{D}$ under some conditions involving the blocks $A, B, C, D$, and the Drazin inverses of $A$ and $D$. Let us recall that the Drazin inverse of any square comples matrix always exists (see e.g., [1, Ch. 4])

First, we will state some auxiliary lemmas. 
Lemma 3.1. (See $\left[1\right.$, Ch. 4] or $\left[2\right.$, Th. 7.8.4]). Let $A \in \mathbb{C}^{m \times n}, B \in \mathbb{C}^{n \times m}$. Then $(A B)^{D}=$ $A\left[(B A)^{D}\right]^{2} B$.

Lemma 3.2. (See [7] or [21]). Let $A \in \mathbb{C}^{m \times n}, B \in \mathbb{C}^{n \times m}$. Then

$$
\left[\begin{array}{cc}
0 & A \\
B & 0
\end{array}\right]^{D}=\left[\begin{array}{cc}
0 & (A B)^{D} A \\
(B A)^{D} B & 0
\end{array}\right] .
$$

Lemma 3.3. (See [2, Ch. 7] or [23]). Let $M_{1}$ and $M_{2}$ be of a form

If $r=\operatorname{ind}(A)$ and $s=\operatorname{ind}(B)$, then

$$
M_{1}=\left[\begin{array}{ll}
A & 0 \\
C & B
\end{array}\right], \quad M_{2}=\left[\begin{array}{cc}
B & C \\
0 & A
\end{array}\right] .
$$

$$
M_{1}^{D}=\left[\begin{array}{cc}
A^{D} & 0 \\
S & B^{D}
\end{array}\right], \quad M_{2}^{D}=\left[\begin{array}{cc}
B^{D} & S \\
0 & A^{D}
\end{array}\right],
$$

where

$$
S=\left[\sum_{i=0}^{r-1}\left(B^{D}\right)^{i+2} C A^{i}\right] A^{\pi}+B^{\pi}\left[\sum_{i=0}^{s-1} B^{i} C\left(A^{D}\right)^{i+2}\right]-B^{D} C A^{D} .
$$

Let $M$ be a $2 \times 2$ block matrix represented as in (8). Let $r=\operatorname{ind}(A)$ and $s=\operatorname{ind}(D)$. To state next lemma, we define the following matrices, being $k$ a nonnegative integer.

$$
\Sigma_{k}=\left(D^{D}\right)^{2} \sum_{i=0}^{r-1}\left(D^{D}\right)^{i+k} C A^{i} A^{\pi}+D^{\pi} \sum_{i=0}^{s-1} D^{i} C\left(A^{D}\right)^{i+k}\left(A^{D}\right)^{2}-\sum_{i=0}^{k}\left(D^{D}\right)^{i+1} C\left(A^{D}\right)^{k-i+1}
$$

Lemma 3.4. (See [17]). Let $M$ be a matrix of a form (8). If $B C=0$ and $B D=0$, then

where $\Sigma_{0}$ and $\Sigma_{1}$ are defined in (10).

$$
M^{D}=\left[\begin{array}{cc}
A^{D} & \left(A^{D}\right)^{2} B \\
\Sigma_{0} & D^{D}+\Sigma_{1} B
\end{array}\right]
$$

Lemma 3.5. Let $X \in \mathbb{C}^{n \times n}$. Then $\left(X^{2} X^{D}\right)^{D}=X^{D},\left(X^{2} X^{D}\right)^{\pi}=X^{\pi}$, and $\operatorname{ind}\left(X^{2} X^{D}\right)=1$.

Proof. The Jordan canonical form of $X$ permits write $X=S(C \oplus N) S^{-1}$, where $S$ and $C$ are nonsingular, and $N$ is nilpotent. Evidently, $X^{D}=S\left(C^{-1} \oplus 0\right) S^{-1}$. Now, it is evident $X^{2} X^{D}=S(C \oplus 0) S^{-1}$, which leads to the affirmations of this lemma.

Using Theorem 2.1 and the previous lemmas, we get the following results.

Theorem 3.1. Let $M$ be given by (8) and let $r=\operatorname{ind}(A)$.

(i) If $A B=B D, D C=C A$, and $B D^{D}=0$, then

$$
\begin{gathered}
M^{D}=\left[\begin{array}{cc}
A^{D} & \left(A^{D}\right)^{2} B \\
\Phi_{0} & D^{D}+\Phi_{1} A A^{D} B
\end{array}\right]+\sum_{i=0}^{r-1}\left[\begin{array}{cc}
0 & (B C)^{D} B \\
(C B)^{D} C & 0
\end{array}\right]^{i}\left[\begin{array}{cc}
(-A)^{i} A^{\pi} & 0 \\
0 & (-D)^{i} D^{\pi}
\end{array}\right], \\
\text { where } \\
\Phi_{0}=\left(D^{D}\right)^{2} C A^{\pi}-D^{D} C A^{D}
\end{gathered}
$$

and

$$
\Phi_{1}=\left(D^{D}\right)^{3} C A^{\pi}-D^{D} C\left(A^{D}\right)^{2}-\left(D^{D}\right)^{2} C A^{D} .
$$


(ii) If $A B=B D, D C=C A$, and $B C=0$, then

$$
M^{D}=\left[\begin{array}{cc}
A^{D} & -\left(A^{D}\right)^{2} B \\
-\left(D^{D}\right)^{2} C & D^{D}+\left(D^{D}\right)^{3} C B
\end{array}\right] .
$$

Proof. (i) We can split the matrix $M$ as $M=P+Q$, where

$$
P=\left[\begin{array}{cc}
A & 0 \\
0 & D
\end{array}\right], \quad Q=\left[\begin{array}{cc}
0 & B \\
C & 0
\end{array}\right] .
$$

From $A B=B D$ and $D C=C A$, we have $P Q=Q P$. Applying Theorem 1.1 and Theorem 2.1 , we get

Observe that

$$
M^{D}=\left(P P^{D}(P+Q)\right)^{D}+\left[\sum_{i=0}^{r-1}\left(Q^{D}\right)^{i+1}(-P)^{i}\right] P^{\pi} .
$$

$$
\left(P P^{D}(P+Q)\right)^{D}=\left[\begin{array}{cc}
A^{2} A^{D} & A A^{D} B \\
D D^{D} C & D^{2} D^{D}
\end{array}\right]^{D} .
$$

From $B D^{D}=0$, the matrix $P P^{D}(P+Q)$ satisfies Lemma 3.4. In view of Lemma 3.5 we get (recall that the index of matrices $A^{2} A^{D}$ and $D^{2} D^{D}$ is 1 )

where

$$
\left(P P^{D}(P+Q)\right)^{D}=\left[\begin{array}{cc}
A^{D} & \left(A^{D}\right)^{2} B \\
\Phi_{0} & D^{D}+\Phi_{1} A A^{D} B
\end{array}\right],
$$

and

$$
\Phi_{0}=\left(D^{D}\right)^{2} C A^{\pi}-D^{D} C A^{D}
$$

Also we have

$$
\Phi_{1}=\left(D^{D}\right)^{3} C A^{\pi}-D^{D} C\left(A^{D}\right)^{2}-\left(D^{D}\right)^{2} C A^{D} .
$$

$$
\sum_{i=0}^{r-1}\left(Q^{D}\right)^{i+1}(-P)^{i}=\sum_{i=0}^{r-1}\left[\begin{array}{cc}
0 & (B C)^{D} B \\
(C B)^{D} C & 0
\end{array}\right]^{i}\left[\begin{array}{cc}
(-A)^{i} & 0 \\
0 & (-D)^{i}
\end{array}\right] .
$$

The proof of (i) is finished.

(ii) Now, we split the matrix $M$ as $M=P+Q$, where

$$
P=\left[\begin{array}{cc}
0 & B \\
C & 0
\end{array}\right], \quad Q=\left[\begin{array}{cc}
A & 0 \\
0 & D
\end{array}\right] .
$$

From $A B=B D$ and $D C=C A$, we have $P Q=Q P$. Hence we can use the expression (11); but now for the matrices $P$ and $Q$ defined in (12).

Since $B C=0$, it is easy to get $P^{3}=0$. Therefore, $P^{D}=0$ and (11) reduces to

$$
M^{D}=Q^{D}-\left(Q^{D}\right)^{2} P+\left(Q^{D}\right)^{3} P^{2} .
$$

Furthermore, we have

and

$$
\left(Q^{D}\right)^{2} P=\left[\begin{array}{cc}
\left(A^{D}\right)^{2} & 0 \\
0 & \left(D^{D}\right)^{2}
\end{array}\right]\left[\begin{array}{cc}
0 & B \\
C & 0
\end{array}\right]=\left[\begin{array}{cc}
0 & \left(A^{D}\right)^{2} B \\
\left(D^{D}\right)^{2} C & 0
\end{array}\right] .
$$

The proof is finished.

$$
\left(Q^{D}\right)^{3} P^{2}=\left[\begin{array}{cc}
\left(A^{D}\right)^{3} & 0 \\
0 & \left(D^{D}\right)^{3}
\end{array}\right]\left[\begin{array}{cc}
0 & 0 \\
0 & C B
\end{array}\right]=\left[\begin{array}{cc}
0 & 0 \\
0 & \left(D^{D}\right)^{3} C B
\end{array}\right] .
$$


Theorem 3.2. Let $M$ be given by (8). If $B C=0, A B D^{D}=0, C A^{\pi} B=0$, and $A B=B D$, then

where $\Sigma_{0}$ and $\Sigma_{1}$ are defined in (10).

$$
M^{D}=\left[\begin{array}{cc}
A^{D} & \left(A^{D}\right)^{2} B \\
\Sigma_{0} & D^{D}+\Sigma_{1} A A^{D} B-D^{D} \Sigma_{0} A^{\pi} B
\end{array}\right],
$$

Proof. We can split the matrix $M$ as $M=P+Q$, where

$$
P=\left[\begin{array}{cc}
0 & A^{\pi} B \\
0 & 0
\end{array}\right], \quad Q=\left[\begin{array}{cc}
A & A A^{D} B \\
C & D
\end{array}\right] .
$$

From $B C=0, C A^{\pi} B=0$, and $A B=B D$ we have $P Q=Q P$. Moreover it is trivial to verify $P^{2}=0$, hence $P^{D}=0$. Applying Theorem 2.1, we get

$$
M^{D}=Q^{D}-\left(Q^{D}\right)^{2} P .
$$

Matrix $Q$ satisfies Lemma 3.4, so we get

$$
Q^{D}=\left[\begin{array}{cc}
A^{D} & \left(A^{D}\right)^{2} A A^{D} B \\
\Sigma_{0} & D^{D}+\Sigma_{1} A A^{D} B
\end{array}\right],
$$

where $\Sigma_{0}$ and $\Sigma_{1}$ are defined in (10). Evidently, $\left(A^{D}\right)^{2} A A^{D} B=\left(A^{D}\right)^{2} B$. Now,

$$
Q^{D} P=\left[\begin{array}{cc}
A^{D} & \left(A^{D}\right)^{2} B \\
\Sigma_{0} & D^{D}+\Sigma_{1} A A^{D} B
\end{array}\right]\left[\begin{array}{cc}
0 & A^{\pi} B \\
0 & 0
\end{array}\right]=\left[\begin{array}{cc}
0 & 0 \\
0 & \Sigma_{0} A^{\pi} B
\end{array}\right]
$$

because $A^{D} A^{\pi}=0$. Therefore,

$$
\left(Q^{D}\right)^{2} P=\left[\begin{array}{cc}
A^{D} & \left(A^{D}\right)^{2} B \\
\Sigma_{0} & D^{D}+\Sigma_{1} A A^{D} B
\end{array}\right]\left[\begin{array}{cc}
0 & 0 \\
0 & \Sigma_{0} A^{\pi} B
\end{array}\right]=\left[\begin{array}{cc}
0 & \left(A^{D}\right)^{2} B \Sigma_{0} A^{\pi} B \\
0 & \left(D^{D}+\Sigma_{1} A A^{D} B\right) \Sigma_{0} A^{\pi} B
\end{array}\right] .
$$

Observe that $A^{D} B D^{D}=\left(A^{D}\right)^{2} A B D^{D}=0$, which leads to

Thus,

$$
\begin{aligned}
A^{D} B \Sigma_{0} & =A^{D} B\left(\left(D^{D}\right)^{2} \sum_{i=0}^{r-1}\left(D^{D}\right)^{i} C A^{i} A^{\pi}+D^{\pi} \sum_{i=0}^{s-1} D^{i} C\left(A^{D}\right)^{i}\left(A^{D}\right)^{2}-D^{D} C A^{D}\right) \\
& =A^{D} B D^{\pi} \sum_{i=0}^{s-1} D^{i} C\left(A^{D}\right)^{i}\left(A^{D}\right)^{2} \\
& =A^{D} B D^{\pi} C\left(A^{D}\right)^{2} \\
& =A^{D} B\left(I-D D^{D}\right) C\left(A^{D}\right)^{2} \\
& =A^{D} B C\left(A^{D}\right)^{2}=0 .
\end{aligned}
$$

$$
\left(Q^{D}\right)^{2} P=\left[\begin{array}{cc}
0 & 0 \\
0 & D^{D} \Sigma_{0} A^{\pi} B
\end{array}\right]
$$

To prove the theorem, it is enough consider (13), (14), and (15).

Next result generalizes Lemma 3.3

Theorem 3.3. Let $M$ be a matrix written as in (8). If $B C=0, C B=0$, and $A B=B D$, then

$$
M^{D}=\left[\begin{array}{cc}
A^{D} & -B\left(D^{D}\right)^{2} \\
S & D^{D}
\end{array}\right]
$$


where

$r=\operatorname{ind}(A)$, and $s=\operatorname{ind}(D)$.

$$
S=\sum_{i=0}^{r-1}\left(D^{D}\right)^{i+2} C A^{i} A^{\pi}+\sum_{i=0}^{s-1} D^{\pi} D^{i} C\left(A^{D}\right)^{i+2}-D^{D} C A^{D},
$$

Proof. We split the matrix $M$ as $M=P+Q$, where

$$
P=\left[\begin{array}{ll}
0 & B \\
0 & 0
\end{array}\right], \quad Q=\left[\begin{array}{ll}
A & 0 \\
C & D
\end{array}\right] .
$$

From the hypotheses of the theorem we get $P Q=Q P$. Since $P^{2}=0$, then $P^{D}=0$ and $P^{\pi}=I$. Thus, Theorem 2.1 and Theorem 1.1 imply

$$
M^{D}=Q^{D}-P\left(Q^{D}\right)^{2} .
$$

By using Lemma 3.3 we can find an expression for $Q^{D}$ :

$$
Q^{D}=\left[\begin{array}{cc}
A^{D} & 0 \\
S & D^{D}
\end{array}\right]
$$

where $S$ is defined in (16). Now we have

$$
P Q^{D}=\left[\begin{array}{cc}
B S & B D^{D} \\
0 & 0
\end{array}\right] \quad \text { and } \quad Q^{D} P=\left[\begin{array}{cc}
0 & A^{D} B \\
0 & S B
\end{array}\right]
$$

By Theorem 1.1, we get $B S=0$ and $S B=0$ (in addition, we get $B D^{D}=A^{D} B$, but this equlity will not be useful). Now,

$$
P\left(Q^{D}\right)^{2}=\left(P Q^{D}\right) Q^{D}=\left[\begin{array}{cc}
B D^{D} S & B\left(D^{D}\right)^{2} \\
0 & 0
\end{array}\right] \quad \text { and } \quad Q^{D}\left(P Q^{D}\right)=\left[\begin{array}{cc}
0 & A^{D} B D^{D} \\
0 & S B D^{D}
\end{array}\right] .
$$

As before, by Theorem 1.1, we get

$$
P\left(Q^{D}\right)^{2}=\left[\begin{array}{cc}
0 & B\left(D^{D}\right)^{2} \\
0 & 0
\end{array}\right]
$$

To prove the theorem, it is enough to consider (17), (18), and (19).

\section{References}

[1] A. Ben-Israel, T.N.E. Greville, Generalized Inverses. Theory and Applications (2nd edition), Springer-Verlag, 2003.

[2] S.L. Campbell, C.D. Meyer, Jr., Generalized Inverses of Linear Transformations, Pitman (Advanced Publishing Program), Boston, MA, 1979, (reprinted by Dover, 1991).

[3] N. Castro-González, J.J. Koliha, Additive perturbation results for the Drazin inverse, Linear Algebra Appl. 397 A (2005), 279-297.

[4] N. Castro-González, E. Dopazo, M.F. Martínez-Serrano, On the Drazin inverse of the sum of two operators and its application to operator matrices, J. Math. Anal. Appl. 350 (2008) 207-215. 
[5] N. Castro-González, M.F. Martínez-Serrano, Expressions for the g-Drazin inverse of additive perturbed elements in a Banach algebra, Linear Algebra Appl. 432 (2010), 1885-1895.

[6] N. Castro-González, J.J. Koliha, New additive results for the Drazin inverse, Proc. Roy. Soc. Edinburgh, 134 A (2004), 1085-1097.

[7] M. Catral, D.D. Olesky, and P. van den Driessche, Block representations of the Drazin inverse of a bipartite matrix, Electron. J. Linear Algebra, 18 (2009), 98-107.

[8] J.L. Chen, G.F. Zhuang, Y. Wei, The Drazin inverse of a sum of morphisms, Acta. Math. Scientia (in Chinese). 29 A (3)(2009), 538-552.

[9] D.S. Cvetković-Ilić, D.S. Djordjević, Y. Wei, Additive results for the generalized Drazin inverse in a Banach algebra, Linear Algebra Appl. 418 (2006), 53-61.

[10] D.S. Cvetković-Ilić, A note on the representation for the Drazin inverse of $2 \times 2$ block matrices, Linear Algebra Appl. 429 (2008) 242-248.

[11] C. Deng, The Drazin inverses of sum and difference of idempotents, Linear Algebra Appl. 430 (2009), 1282-1291.

[12] C. Deng, Y. Wei, Characterizations and representations of the Drazin inverse of idempotents, Linear Algebra Appl. 431 (2009), 1526-1538.

[13] C. Deng, Y. Wei, New additive results for the generalized Drazin inverse, J. Math. Anal. Appl. 370 (2010), 313-321.

[14] D.S. Djordjević, P.S. Stanimirović, On the generalized Drazin inverse and generalized resolvent, Czechoslovak Math. J. 51(126)(2001) 617-634.

[15] D.S. Djordjević, Y. Wei, Additive results for the generalized Drazin inverse, J. Aust. Math. Soc. 73 (2002), 115-125.

[16] D.S. Djordjević, V. Rakočević, Lectures on Generalized inverses, University of Niš (2008).

[17] E. Dopazo, M.F. Martínez-Serrano, Further results on the representation of the Drazin inverse of a $2 \times 2$ block matrices, Linear Algebra Appl. 432 (2010), 1896-1904.

[18] M.P. Drazin, Pseudo-inverses in associative rings and semiproup, Amer. Math. Monthly. 65 (1958), 506-514.

[19] R.E. Hartwig, G.R. Wang, and Y. Wei, Some additive results on Drazin inverse, Linear Algebra Appl. 322 (2001), 207-217.

[20] R.E. Hartwig, X. Li, Y. Wei, Representations for the Drazin inverse of a $2 \times 2$ block matrix, SIAM J. Matrix Anal. Appl. 27 (2006) 757-771. 
[21] Y. Liu, C.G. Cao, Drazin inverse for some partitioned matrices over skew fields, Journal of Natural Science of Hei Long Jiang University. 24 (2004), 112-114.

[22] J. Ljubisavljević, D. S. Cvetković-Ilić, Additive results for the Drazin inverse of block matrices and applications, J. Comput. Appl. Math. 235 (2011) 3683-3690.

[23] C.D. Meyer Jr., N.J. Rose, The index and the Drazin inverse of block triangular matrices, SIAM J. Appl. Math. 33 (1) (1977), 1-7.

[24] L. Wang, H.H. Zhu, X. Zhu, J.L. Chen. Additive property of Drazin invertibility of elements, arXiv: 1307.1816v1 [math.RA], 15 july (2013).

[25] H. Yang, X. Liu, The Drazin inverse of the sum of two matrices and its applications, J. Comput. Appl. Math. 235 (2011) 1412-1417.

Xiaoji Liu: Faculty of Science, Guangxi University for Nationalities, Nanning 530006, P.R. China

Email: xiaojiliu72@126.com

Xiaolan Qin Faculty of Science, Guangxi University for Nationalities, Nanning 530006, P.R. China

Julio Benítez Universidad Politécnica de Valencia, Instituto de Matemtica Multidisciplinar, Valencia 46022, Spain

Email: jbenitez@mat.upv.es 